\title{
Laboratory study on sediment diffusion and deposition into blind channels
}

\author{
Qingquan Liu * \\ Institute of Mechanics, Chinese Academy of Sciences, 15, Beisihuanxi Road, Beijing 100080, China
}

Received 15 June 2004; received in revised form 9 December 2005; accepted 3 January 2006

Available online 2 March 2006

\begin{abstract}
Any waterway with one end closed and the other open is generally called a blind channel. The main flow tends to expand, separate, and cause circulation at the mouth of blind channels. The main flow continuously transfers momentum and sediment into the circulation region through the turbulent mixing region (TMR) between them, thus leading to a large amount of sediment deposition in the blind channels. This paper experimentally investigated the properties of the water flow and sediment diffusion in TMR, demonstrating that both water flow and sediment motion in TMR approximately coincide with a similar structure as in the free mixing layer induced by a jet. The similarity functions of flow velocity and sediment concentration are then assumed, based on observation, and the resulting calculation of these functions is substantially facilitated. For the kind of low velocity flow system of blind channels with a finite width, a simple formula for the sediment deposition rate in blind channels is established by analyzing the gradient of crosswise velocity and sediment concentration in TMR.

(C) 2006 Elsevier Ltd. All rights reserved.
\end{abstract}

Keywords: Sediment diffusion; Sediment deposition; Turbulent mixing; Similarity solution; Circulation flow; Blind channel

\section{Introduction}

Channels with one end closed and the other open are often found in harbor basins, waterways leading to navigation locks, trench intakes with the gate closed, etc. This kind of waterway is generally called a blind channel. The velocity of water flow in this kind of blind channels is commonly low, compared with the main flow of the adjacent river, thus a large amount of the sediment drawn into blind channels very likely would deposit there. Sediment deposition in blind channels has been observed in many engineering projects concerned with navigation problems at the water intakes located along rivers and beaches $[5,10,11]$. For instance, the sedimentation rate in the harbor basins of Hamburg, Germany, is on the order of $2 \times 10^{6} \mathrm{~m}^{3} /$ year. Every year, approximately $1 \times 10^{5} \mathrm{~m}^{3}$ sediment accumulates inside the port of Kanazawa located near sand bea-

\footnotetext{
${ }^{*}$ Tel.: +86 10 62554125; fax: +86 1062561284 .

E-mail address: qqliu@imech.ac.cn
}

ches of Japan. Sedimentation lessens the water depth of the harbor, so costly regular dredging is necessary to guarantee safe navigation. Consequently, the prediction of the sediment deposition inside blind channels is of primary significance.

Some researchers have studied the mechanism of sediment deposition and corresponding measures in order to prevent sediment depositing in blind channels. Hangen and Dhanak [3] investigated the momentum and mass exchanges in a rectangular cavity. $\mathrm{Xu}$ [12] obtained a formula to estimate the critical diameter for sediment deposition using experimental data. Xie and Yin [11] established a semi-experiential formula to estimate the rate of sediment deposition by using field data, and applied the formula to the waterway of Gezhouba Dam in Yangtze River. Yue [13] experimentally examined the process of sediment depositing in a cavity flume, and suggested that the circulation flow located at the mouth of blind channels has an average sediment carrying capacity — one factor influencing the sediment deposition in blind channels. Liu [7] estab- 


\begin{tabular}{|c|c|}
\hline \multicolumn{2}{|c|}{ Notation } \\
\hline$a$ & experical coefficient \\
\hline$a_{1}$ & integral constant \\
\hline$a_{2}$ & integral constant \\
\hline$a_{3}$ & integral constant \\
\hline$b$ & width of the turbulent mixing region \\
\hline$B_{\mathrm{m}}$ & width of the main flow \\
\hline$B_{0}$ & width of the blind channel \\
\hline$c$ & constant \\
\hline$c_{1}$ & constant \\
\hline$f(\zeta)$ & similarity function of stream function \\
\hline$F(\zeta)$ & similarity function of sediment concentration \\
\hline$g$ & gravity acceleration \\
\hline$g_{\mathrm{s}}$ & $\begin{array}{l}\text { sediment diffusion rate of unit area crossing the } \\
\text { interface between the main flow and the circula- } \\
\text { tion flow }\end{array}$ \\
\hline$G_{\mathrm{s}}$ & $\begin{array}{l}\text { sediment amounts entering into the blind chan- } \\
\text { nel per unit time }\end{array}$ \\
\hline$H$ & depth of water flow \\
\hline$K$ & empirical parameter \\
\hline$L$ & length of the blind channel \\
\hline$l_{\mathrm{s}}$ & mixing length \\
\hline$m$ & index number \\
\hline$p$ & water pressure \\
\hline$S$ & sediment concentration of water flow in TMR \\
\hline$S_{\mathrm{m}}$ & sediment concentration of the main flow \\
\hline$S_{\mathrm{r}}$ & $\begin{array}{l}\text { averaged sediment concentration of the circula- } \\
\text { tion flow }\end{array}$ \\
\hline$S_{\mathrm{r}^{*}}$ & $\begin{array}{l}\text { saturated sediment carrying capacity of the cir- } \\
\text { culation flow }\end{array}$ \\
\hline
\end{tabular}

$u \quad$ flow velocity component in the $x$-direction

$U_{\mathrm{m}} \quad$ averaged velocity of the main flow

$u^{\prime} \quad$ turbulent velocity components in the $x$-direction

$v^{\prime} \quad$ turbulent velocity components in the $y$-direction

$v$ flow velocity component in the $y$-direction

$\alpha \quad$ deflection angle between the main-circulation flow interface and the geometrical interface outer diffusive angle of TMR inner diffusive angle of TMR coefficient empirical parameter turbulent viscosity at the interface between the main flow and the circulation flow sediment diffusion coefficient in the $y$-direction the specific gravity of water the specific gravity of sediment particles water viscosity turbulent viscosity in TMR density of water fall velocity of sediment particle stream function first order similarity solution of stream function similarity variable value of similarity variable in outer boundary of TMR value of similarity variable in inner boundary of TMR lished a formula to calculate the sediment carrying capacity of circulation flow from theory and experiments. Liu [8] also reported a systemic experimental study, including the distributions of flow velocity and sediment concentration, the hydraulic sorting of sediment particles, and the quantity and patterns of sediment deposition in the circulation flow as well. Experimental results of Altai et al. [1] have clearly shown the existence of a core region in the circulation flow near a cavity mouth where the retention time is significantly greater than the outer region surrounding the core. This finding is helpful in understanding the pattern of sediment deposition in blind channels. The methods for preventing sediment deposition in blind channels also have received more attention in recent years [4,9]. Hofland et al. [4] found that a deflecting wall is able to reduce the sedimentation of river harbor under tidal conditions. In addition, some mathematical models were applied to investigate the sediment deposition in blind channels. For example, Dong [2] established a 2-D depth-averaged flow and sediment diffusion model to simulate the sediment transport in a blind channel. These investigations have exhibited a rather clear pattern of sediment transport and deposition in blind channels. However, these previous studies did not pay close attention to modeling the flow and sediment transport in the turbulent mixing region (TMR) between the main flow and the circulation in blind channels, which, as a matter of fact, is one of the most significant features affecting sediment deposition in blind channels.

Generally speaking, the flow system of blind channels could be divided into at least three regions: (1) main flow region, (2) circulation flow region deep into the blind channel, and (3) turbulent mixing region between the main flow and the circulation regions. For long blind channels, there exists the forth region - the upper end of the system beyond the circulation zone. Sometimes, the fourth region is called the density flow region because the density flow commonly occurs there. The interaction between the main flow and the circulation flow is controlled by the water flow in TMR, where turbulent mixing, namely momentum and mass exchanges constantly take place. For sediment-laden flow, the main flow and the circulation flow also exchange sediment through TMR. Both the flow velocity and the turbulence intensity in blind channels are usually low enough such that the sediment carrying capacity of flow in the circulation region is far less than that in the main flow. As a result, the sediment coming from the main flow may largely 
deposit in the blind channels $[11,13]$. According to the sediment diffusion law in water flow, the crosswise sediment diffusion rate in TMR, $g_{\mathrm{s}}$, can be expressed as

$g_{\mathrm{s}}=\varepsilon_{\mathrm{s} y} \frac{\partial S}{\partial y}$.

Introducing Prandtl's mixing length hypothesis, $\varepsilon_{\mathrm{s} y}=l^{2}(\partial u /$ $\partial y$ ), leads to the following expression:

$g_{\mathrm{s}}=l^{2} \frac{\partial u}{\partial y} \frac{\partial S}{\partial y}$

where $S$ is the sediment concentration of flow, $\varepsilon_{s y}$ is the sediment diffusion coefficient in the $y$-direction, $l$ is the mixing length, $u$ is the longitudinal depth-averaged velocity of flow. Assuming all sediment diffusing from the main flow will deposit in the blind channel, the sediment deposition rate can be estimated by using Eq. (2) at the interface between the main flow and the blind channel $(y=0)$.

From Eq. (2), it is observed that the sediment diffusion from the main flow to the blind channel is mainly dependent on the crosswise gradients of the sediment concentration and flow velocity at the interface in TMR. Thus, the sediment deposition rate in blind channels is governed by turbulent diffusion in TMR. To deeply analyze the fluid motion and sediment diffusion in TMR is a fundamental for understanding the sediment transport process and estimating the deposition rate in blind channels. Thus, the purpose of the current study is to present the law of fluid motion and sediment diffusion in TMR on the basis of experimental data and analytical solutions, sequentially establishing a simple theoretical formula in order to estimate the sediment deposition rate in blind channels.

\section{Hydraulic experiments}

\subsection{Description of experiments}

A set of experiments were conducted in the State Key Laboratory of Water Resources and Hydropower Engineering Science at Wuhan University, China. The experimental set up consists of a main flume of rectangular cross section and a blind flume perpendicular to the former

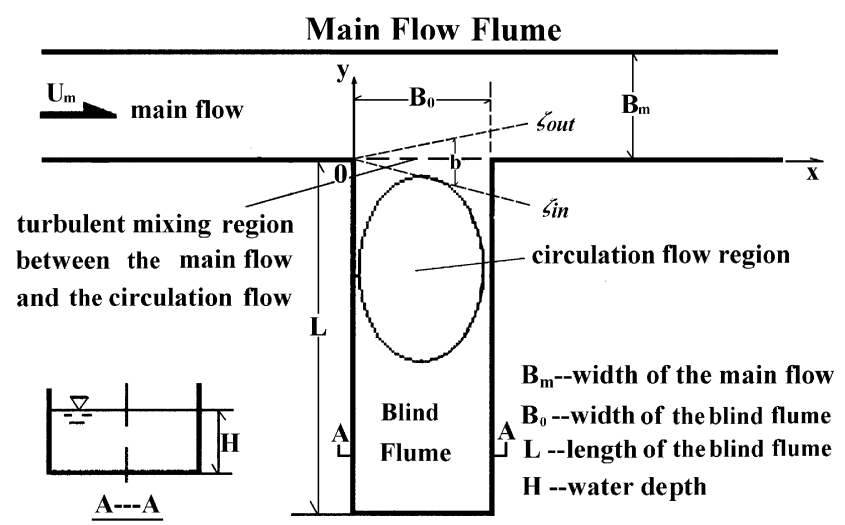

Fig. 1. Plan sketch of circulation flow system in a blind channel. as shown in Fig. 1. The main flume is $1500 \mathrm{~cm}$ long, $80 \mathrm{~cm}$ wide, and $40 \mathrm{~cm}$ deep, the bed slope of the flume is about $0.1 \%$. The blind flume is connected to the main flume in the middle of the main flume, $900 \mathrm{~cm}$ downstream from the entrance. The length and width of the blind flume can be adjusted according to experimental needs with a maximum length of $200 \mathrm{~cm}$ and width of $80 \mathrm{~cm}$. The bottoms of the main flume and the blind flume are made of concrete, and the walls of both are aluminium board. The water in the experiments was recycled by passing a cylinder reservoir, and could be adequately agitated in the reservoir for turbid water experiments.

A triangular-notch weir and a Venturi flowmeter were used to measure discharges of clear and turbid water flows, respectively. The flow velocity was measured by a tachometer with a small propeller $8 \mathrm{~mm}$ in diameter, which can measure even a small velocity of about $2.4 \mathrm{~cm} / \mathrm{s}$. The average value at three points $(0.2 H, 0.6 H, 0.8 H$, where $H$ is the water depth) on a vertical profile was used to represent the depth-averaged velocity, while the vertical average sediment concentration was obtained by sampling at five elevations of $0.1 \mathrm{H}, 0.2 \mathrm{H}, 0.4 \mathrm{H}, 0.6 \mathrm{H}$, and $0.8 \mathrm{H}$. The sediment deposition amount in the blind channel was measured by weighting all sediment in the blind channel. Namely, when a set of experiment was finished, we first closed the mouth of the blind channel, and then drained out the water in the blind channel. At last, all sediment was sampled, dried, and weighted to obtain the sediment deposition amount. Nonuniform plastic sand of specific weight $\gamma_{\mathrm{s}}=1.05 \mathrm{t} / \mathrm{m}^{3}$ and median diameter $d_{50}=0.33 \mathrm{~mm}$ was used in the turbid water experiments.

Six different cases were carried out to observe the distributions of depth-averaged velocity, sediment concentration, flow velocity and sediment concentration gradients in TMR, respectively. Twelve different experimental cases were also conducted to observe the sediment deposition rate in the blind channel. The summary of experimental cases is shown in Table 1.

\subsection{General characteristics of the flow in TMR}

The flume test results of the depth-averaged velocity distribution are plotted in Fig. 2, where $H$ is the water depth, $U_{\mathrm{m}}$ is the mean depth-averaged velocity of the main flow, $u$ is the depth-averaged longitudinal velocity of flow, and $B_{0}$ is the width of the blind channel. (the Reynolds number of the main flow is $R e=26,670)$. Experimental results show that the circulation flow located at the mouth of the blind channel exerts a retarding action on the main flow through the flow in TMR. As a matter of fact, because of the wall resistance, the depth-averaged velocity distribution should not be uniform along across direction of the main flow. The velocity generally reduces near the walls, and the vertical profile of velocity also exhibits different patterns. However, due to the limitation of the instrumentation used in the experiments and the method of observation, some of the wall boundary layer effect may be lost by depth averag- 
Table 1

The summary of experimental conditions for different observations

\begin{tabular}{|c|c|c|c|c|c|c|c|}
\hline \multirow[t]{2}{*}{ Run } & \multirow{2}{*}{ Fluid type } & \multicolumn{3}{|c|}{ Main flow } & \multicolumn{2}{|c|}{ Blind flume } & \multirow[t]{2}{*}{ The specific items of observation } \\
\hline & & $\begin{array}{l}\text { Flow } \\
\text { velocity } \\
(\mathrm{cm} / \mathrm{s})\end{array}$ & $\begin{array}{l}\text { Water } \\
\text { depth } \\
(\mathrm{cm})\end{array}$ & $\begin{array}{l}\text { Sediment } \\
\text { concentration } \\
\left(\mathrm{kg} / \mathrm{m}^{3}\right)\end{array}$ & $\begin{array}{l}\text { Width } \\
(\mathrm{cm})\end{array}$ & $\begin{array}{l}\text { Length } \\
(\mathrm{cm})\end{array}$ & \\
\hline 1 & Clear water & 37.3 & 12.2 & - & 80 & 200 & The depth-averaged velocity distribution of the main flow \\
\hline 2 & Clear water & 48.0 & 12.5 & - & 80 & 200 & The depth-averaged velocity distribution in TMR \\
\hline 3 & Clear water & 48.9 & 14.5 & - & 80 & 200 & The velocity gradient on the geometrical interface \\
\hline 4 & Water-sediment & 44.6 & 15.0 & 7.56 & 80 & 200 & The sediment concentration distribution of the main flow \\
\hline 7 & Water-sediment & 58.8 & 13.6 & 4.71 & 52 & 200 & The sediment deposition rate in the blind flume \\
\hline 8 & Water-sediment & 54.6 & 13.5 & 6.08 & 52 & 200 & The sediment deposition rate in the blind flume \\
\hline 9 & Water-sediment & 43.7 & 13.7 & 5.20 & 52 & 200 & The sediment deposition rate in the blind flume \\
\hline 10 & Water-sediment & 43.7 & 13.9 & 5.15 & 52 & 200 & The sediment deposition rate in the blind flume \\
\hline 11 & Water-sediment & 44.8 & 13.6 & 10.50 & 52 & 200 & The sediment deposition rate in the blind flume \\
\hline 12 & Water-sediment & 44.2 & 13.8 & 12.31 & 52 & 200 & The sediment deposition rate in the blind flume \\
\hline 17 & Water-sediment & 44.9 & 13.7 & 8.69 & 41 & 200 & The sediment deposition rate in the blind flume \\
\hline 18 & Water-sediment & 44.5 & 13.7 & 8.58 & 31 & 200 & The sediment deposition rate in the blind flume \\
\hline
\end{tabular}

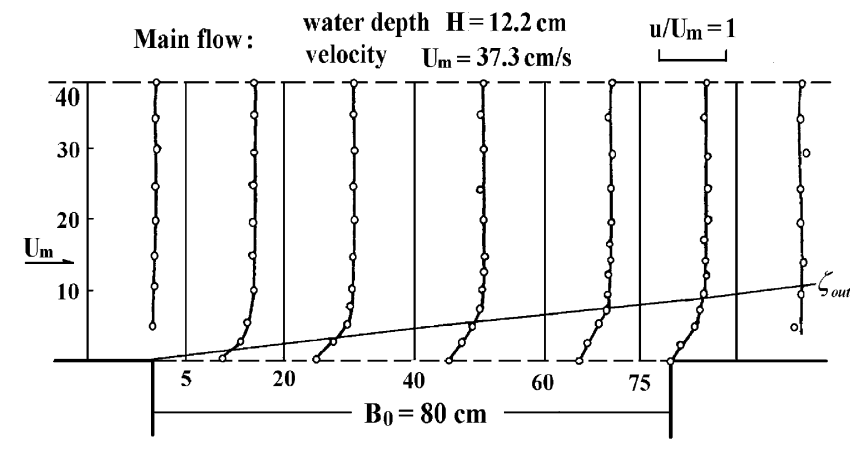

Fig. 2. Distribution across the width of the main flume of depth-averaged longitudinal velocity near the mouth of the blind channel.

ing, which results in the velocities which besides the small region near the walls are approximately uniform. Therefore, the distinct velocity decreases shown in experimental results was mainly caused by the retarding action of the circulation flow located at the mouth of the blind channel, and the momentum deficit region with reduced velocities has a rather clear boundary. From this boundary the depth-averaged velocity of flow in the main flume decreases gradually. Assuming the main flow will transform to TMR flow from this boundary, this line can be defined as a boundary between TMR and main flow (outer boundary). The flow in TMR provides momentum to maintain the steady motion of the circulation flow near the mouth of the blind flume. Conversely, it also retards the main flow, and the affected region gradually increases with the distance downstream.

Therefore, the flow in TMR can be approximately modeled as a free jet near the mouth of blind channels. Actually, experiments show that the generation and development of vortices in TMR are rather intermittent, so the velocity, direction, and pressure of the water flow are always fluctuating, and the boundaries of TMR at both sides also are unstable. But from a time-averaged viewpoint, TMR has relatively clear, fixed boundaries as shown in Fig. 3. There, $\alpha_{1}$ and $\alpha_{2}$ are the outer diffusive angle and the inner diffusive angle of TMR, respectively; $\varsigma_{\text {out }}$ and $\varsigma_{\text {in }}$ are the outer boundary and the inner boundary of TMR, respectively; and $b$ is the width of TMR.

For a turbulent mixing layer with free boundary, its width grows linearly. That is to say, the width of the mixing region, $b$, is generally proportional to the distance from the separation point.

$b=c x$,

where $x$ is the distance from the separation point, $c$ is a constant coefficient.

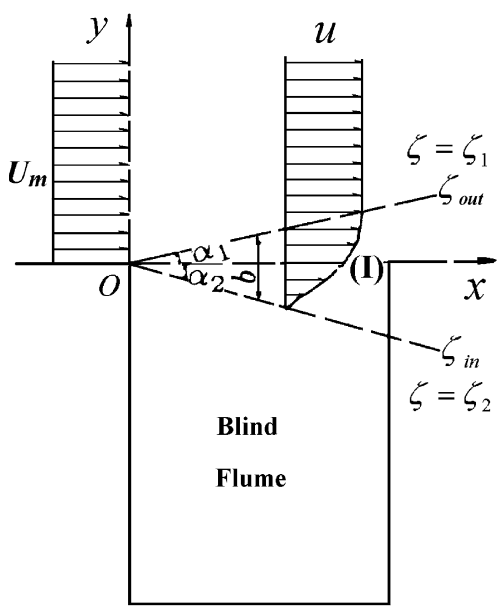

(I) Turbulent Mixing Region

Fig. 3. Plan sketch of the TRM. 
In regard to the TMR flow, its width can still be approximated by Eq. (3) although the flow is disturbed by the downstream wall of blind channel. Of course, the actual boundaries of TMR define an irregular section composed of eddies and exterior flow. Hence, the boundaries expressed by Eq. (3) are only regarded as statistically averaged over time. According to experiments, this intersecting line was obtained as $y=0.107 x$. Consequently, the angle between the outer boundary of TMR and the geometrical interface of the blind channel mouth takes about $6.11^{\circ}$ $\left(=\tan ^{-1} 0.107\right)$. As a matter of fact, the velocity and depth of the main flow may have a little influence on the diffusion angle of TMR. However, the influence is unconspicuous and negligible for low velocity flows according our experiments.

\section{Flow velocity field in TMR and its solution}

\subsection{Mathematical description of the fluid motion in TMR}

Neglecting the influence of sediment on water flow, the velocity distribution in sediment-laden flows could be approximated to possess the same structure as in clear water flow. Due to the sufficient turbulence of the flow in TMR, the problem can be simplified to a plane shallow water problem. The width of TMR is far smaller than the widths of blind channel and the main flow. The water flow in TMR can be considered as a free boundary layer flow. Taking the coordinate system as shown in Fig. 3, and applying the boundary layer theory, the two-dimensional (2-D) equations of the water flow in TMR are simplified as follows:

$\frac{\partial u}{\partial t}+\frac{\partial u u}{\partial x}+\frac{\partial u v}{\partial y}+\frac{\partial \overline{u^{\prime 2}}}{\partial x}+\frac{\partial \overline{u^{\prime} v^{\prime}}}{\partial y}=v \frac{\partial^{2} u}{\partial y^{2}}-\frac{1}{\rho} \frac{\partial p}{\partial x}$,

$\frac{\partial u}{\partial x}+\frac{\partial v}{\partial y}=0$

where $u$ and $v$ are the time-averaged velocity components in the $x$ - and $y$-direction, respectively; $u^{\prime}$ and $v^{\prime}$ are the turbulent velocity components in the $x$ - and $y$-direction; $v$ is the kinematic viscosity of water; $\rho$ is the density of water; and $p$ is the time-averaged pressure.

In the above equations, the lateral motion equation of flow (in the $y$-direction) was neglected. Applying the boundary layer theory [6], through the order of comparison the lateral equation can be simplified as $\partial p / \partial y=0$. That is to say, there is no transverse pressure gradient in the boundary layer (TMR). In other words the pressure in the boundary layer (TMR) is equal to the pressure in the main flow and is a given function of $x$ for the purpose of solving the boundary-layer problem. Thus, the lateral motion equation (in the $y$-direction) need not to be written. Consequently, the equations of the flow in TMR could be simplified as Eqs. (4) and (5).

Suppose the flow in TMR is similar to the flow in a free shear layer and the wall influences could then be not taken into account. The pressure gradient in the $x$-direction also appears to be negligible, i.e., $\partial p / \partial x \approx 0$. The velocity gradient and turbulent velocity in the $x$-direction is far less than those in the $y$-direction, so the term of $\partial \overline{u^{\prime 2}} / \partial x$ can be omitted. Therefore for 2-D steady flow, the flow equation of TMR can be further simplified as follows:

$u \frac{\partial u}{\partial x}+v \frac{\partial u}{\partial y}+\frac{\partial}{\partial y}\left(-\overline{u^{\prime} v^{\prime}}\right)=0$.

Applying Prandtl's mixing length theory, one has

$-\overline{u^{\prime} v^{\prime}}=l^{2}\left|\frac{\partial \bar{u}}{\partial y}\right| \frac{\partial \bar{u}}{\partial y}$.

Assuming that the mixing length $l$ is proportional to the width of TMR, combining Eq. (3) yields:

$l=c c_{1} x \quad\left(c, c_{1}\right.$ are constants $)$.

Consequently, Eq. (6) can be finally written as

$u \frac{\partial u}{\partial x}+v \frac{\partial u}{\partial y}=2 c^{2} c_{1}^{2} x^{2} \frac{\partial u}{\partial y} \frac{\partial^{2} u}{\partial y^{2}} \quad\left(\frac{\partial u}{\partial y}>0\right)$.

Introducing the stream function $\psi$, with $u=\partial \psi /$ $\partial y, \quad v=-\partial \psi / \partial x$, and substituting them into Eq. (9), one obtains

$\frac{\partial \psi}{\partial y} \frac{\partial^{2} \psi}{\partial x \partial y}-\frac{\partial \psi}{\partial x} \frac{\partial^{2} \psi}{\partial y^{2}}=2 c^{2} c_{1}^{2} x^{2} \frac{\partial^{2} \psi}{\partial y^{2}} \frac{\partial^{3} \psi}{\partial y^{3}}$.

The experiments reported in this paper demonstrate that both water flow and sediment motion in TMR approximately coincide with a similar structure as in the free mixing layer induced by a jet. Therefore, the similarity theory can be used to solve this problem. Applying similarity assumption, the partial differential equation (10) may be translated an ordinary differential equation that can be solved analytically.

Supposing $\psi_{0}=U_{\mathrm{m}} y$ ( $U_{\mathrm{m}}$ is the mean depth-averaged velocity of the main flow) is the first-order similarity solution of Eq. (10), and introducing the similarity variable $\zeta=\delta y / x$ ( $\delta$ is an empirical parameter), $\psi_{0}$ may be written as $\psi_{0}=\delta^{-1} U_{\mathrm{m}} x \zeta$.

Assuming the similarity function of the stream function is $f(\zeta)$, then

$\psi=\delta^{-1} U_{\mathrm{m}} x f(\zeta)$.

Substituting Eq. (11) into Eq. (10) and rearranging them, one has

$2 c^{2} c_{1}^{2} \delta^{3} f^{\prime \prime}(\zeta) f^{\prime \prime \prime}(\zeta)+f(\zeta) f^{\prime \prime}(\zeta)=0$.

The previous analysis shows that the partial differential equation (9) can be translated into an ordinary differential equation by means of a similar variable $\zeta=\delta y / x$, where $\delta$ is an empirical coefficient. Letting $\delta=\left(c c_{1}\right)^{-2 / 3}$, Eq. (12) can be further simplified as

$2 f^{\prime \prime}(\zeta) f^{\prime \prime \prime}(\zeta)+f(\zeta) f^{\prime \prime}(\zeta)=0$.

Setting $f^{\prime \prime}(\zeta)=0$ indicates wrongly that there is no velocity gradient in TMR, this is not the actual situation. So 
$f^{\prime \prime}(\zeta)=0$ is not the solution to the problem. Therefore, the similarity function equation of the flow in TMR has the following final form:

$2 f^{\prime \prime \prime}(\zeta)+f(\zeta)=0$.

\subsection{Similarity solution of the water flow in TMR}

Ignoring the influence of the downstream wall of the blind channel, the boundary conditions of Eq. (10) can be specified at the outer boundary of TMR as follows (see Fig. 3): $\partial \psi / \partial y=U_{\mathrm{m}}, \partial^{2} \psi / \partial y^{2}=0$, and $\partial \psi / \partial x=0$ at the outer edge $\zeta_{1}$.

Due to the existence of the circulation flow near the mouth of blind channels, it is not easy to determine the inner boundary condition of the flow in TMR. Considering that the average velocity of the circulation flow is much lower than that of the main flow, and the longitudinal velocity at the inner boundary of TMR is very small, $\partial \psi /$ $\partial y \approx 0$, and $\partial^{2} \psi / \partial y^{2} \approx 0$ at the inner boundary. Thus, the dimensionless boundary conditions of the similarity function of Eq. (3) can be reduced to:

At the outer boundary $\zeta=\zeta_{1}$

$F^{\prime}(\zeta)=1$,

$F^{\prime \prime}(\zeta)=0$,

$F(\zeta)=\zeta_{1}$

At the inner boundary $\zeta=\zeta_{2}$

$F^{\prime}(\zeta)=0$,

$F^{\prime \prime}(\zeta)=0$.

Since the characteristic roots of Eq. (14) are

$r_{1}=2^{-\frac{1}{3}} \mathrm{e}^{\mathrm{i} \frac{\pi}{3}}, \quad r_{2}=2^{-\frac{1}{3}} \mathrm{e}^{\mathrm{i} \pi}, \quad r_{3}=2^{-\frac{1}{3}} \mathrm{e}^{-\mathrm{i} \frac{\pi}{3}}$.

The general solution of Eq. (14) yields the following form:

$f(\zeta)=a_{1} \mathrm{e}^{-A \zeta}+a_{2} \mathrm{e}^{B \zeta} \cos (\sqrt{3} B \zeta)+a_{3} \mathrm{e}^{B \zeta} \sin (\sqrt{3} B \zeta)$,

where $A=2^{-\frac{1}{3}}, B=2^{-\frac{4}{3}}$, and $a_{1}, a_{2}$, and $a_{3}$ are the integral constants determined by the boundary conditions.

By using Eq. (17), a group of nonlinear equations were derived from the boundary conditions (15), (16), and the values of $a_{1}, a_{2}, a_{3}, \zeta_{1}$, and $\zeta_{2}$ can be numerically obtained as follows:

$a_{1}=-0.019, \quad a_{2}=0.185, \quad a_{3}=0.850$,

$\zeta_{1}=1.208, \quad \zeta_{2}=-2.403$.

Consequently, the similarity function of the problem looks like:

$f(\zeta)=-0.019 \mathrm{e}^{-A \zeta}+0.185 \mathrm{e}^{B \zeta} \cos (\sqrt{3} B \zeta)+0.85 \mathrm{e}^{B \zeta} \sin (\sqrt{3} B \zeta)$.

Therefore, the stream function and the velocity field in TMR are $\psi(\zeta)=\delta^{-1} U_{\mathrm{m}} x f(\zeta)$,

$u=U_{\mathrm{m}} f^{\prime}(\zeta)$,

$v=\delta^{-1} U_{\mathrm{m}}\left[\zeta f^{\prime}(\zeta)-f(\zeta)\right]$,

where $\delta=\left(c c_{1}\right)^{-2 / 3}$ is an empirical parameter. Based on the experimental observations, the separation angle between the outer boundary of TMR and the geometrical interface of the blind channel mouth, i.e., the outer diffusive angle of TMR is

$\alpha_{1}=6.11^{\circ}$.

That is $y / x=\tan \alpha_{1}=0.107$, and the outer boundary $\zeta_{1}=\delta y / x=1.208$. Therefore, one has

$\delta=\left(c c_{1}\right)^{-\frac{2}{3}}=11.29$.

In addition, the other diffusive angles (the inner diffusive angle) of TMR can be obtained based on the value of $\zeta_{2}$ as follows:

$\alpha_{2}=-12.02^{\circ}$.

Actually, the interface between the main flow and the circulation flow is not same as the geometrical boundary of the mouth of blind channels, but a streamline of TMR flow, namely, the plane $\psi=0$, corresponding to $\zeta_{0}=-0.27$ or $y / x=-0.024$. As a result, the deflection angle between the actual interface and the geometrical interface is

$\alpha=\arctan (y / x)=-1.37^{\circ}$.

According to the solution of the velocity field, the distribution of the longitudinal velocity of the flow in TMR was numerically calculated. The numerical results (solid line) showed a good agreement with experimental data (dots) for a Reynolds number of the main flow of 34,950 as shown in Fig. 4.

The gradient of the longitudinal velocity $(u)$ along the crosswise $(y)$ direction in TMR is

$\frac{\partial u}{\partial y}=\delta U_{\mathrm{m}} x^{-1} f^{\prime \prime}(\zeta)$

at the interface $\zeta=0$,

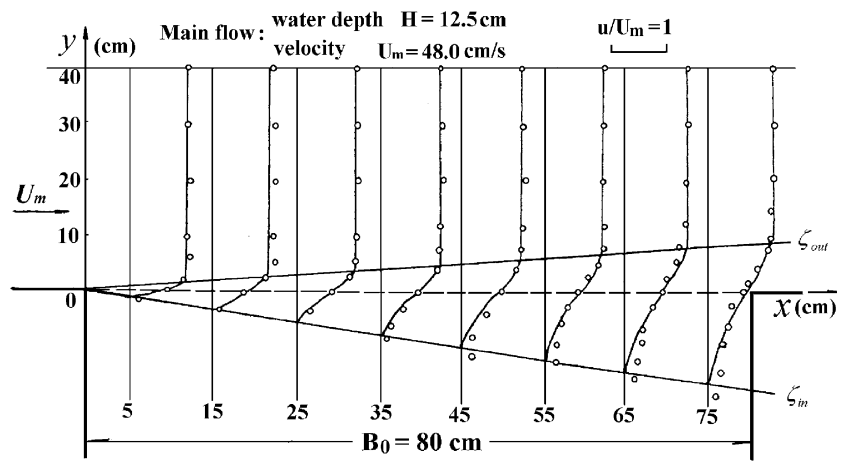

Fig. 4. Distribution of the depth-averaged longitudinal velocity of the flow in TRM where the solid lines are numerical results and circles are experimental measurements for $R e=34,950$. 


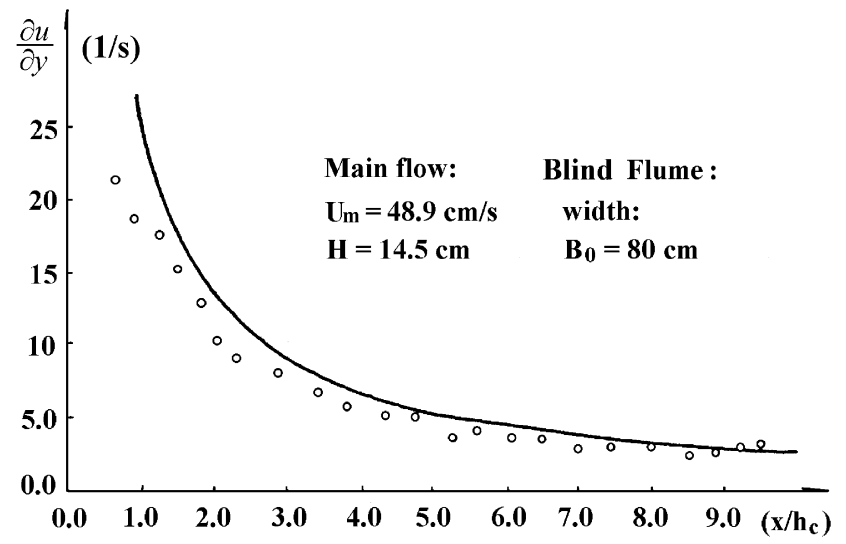

Fig. 5. Velocity gradient on the geometrical interface computed from Eq. (27) (solid line) and experimentally measured.

$$
\left(\frac{\partial u}{\partial y}\right)_{\zeta=0}=4.44 U_{\mathrm{m}} x^{-1}
$$

Taking $(\partial u / \partial y)_{\zeta=0}$ for the ordinate and $\left(x / H_{\mathrm{c}}\right)$ for the abscissa (where $H_{\mathrm{c}}$ is the critical water depth of the main flow, $H_{\mathrm{c}}=\sqrt[3]{\left.\left(U_{\mathrm{m}} H\right)^{2} / g\right)}$, comparison of computational results of Eq. (27) and experimental data is shown in Fig. 5. The experimental value of the velocity gradient was obtained by computing the average variation of velocity between two points near the interface. These two points are located in two sides of interface with a distance of $1 \mathrm{~cm}$, respectively. Thus, the experimental results are a little smaller than the numerical values. Nevertheless, this comparison still shows rather good agreements.

Applying Prandtl's momentum transmission theory, the turbulent viscosity of flow in TMR can be obtained as

$v_{\mathrm{t}}=l^{2} \frac{\partial u}{\partial y}=\delta^{-2} U_{\mathrm{m}} x f^{\prime \prime}(\zeta)$.

Therefore, the turbulent viscosity along the interface between the main flow and the circulation $(\zeta=-0.27)$ could be expressed as follows:

$\varepsilon_{y}=0.00325 U_{\mathrm{m}} x$.

\section{Sediment diffusion equation and its solution}

\subsection{Sediment diffusion equation in $T M R$}

When ignoring molecular diffusion, the diffusion equation of sediment movement in TMR takes the following form:

$$
\begin{aligned}
\frac{\partial S}{\partial t} & +u \frac{\partial S}{\partial x}+v \frac{\partial S}{\partial y}+w \frac{\partial S}{\partial z}-\omega \frac{\partial S}{\partial z} \\
& =\frac{\partial}{\partial x}\left(\varepsilon_{s x} \frac{\partial S}{\partial x}\right)+\frac{\partial}{\partial y}\left(\varepsilon_{s y} \frac{\partial S}{\partial y}\right)+\frac{\partial}{\partial z}\left(\varepsilon_{\mathrm{s} z} \frac{\partial S}{\partial z}\right),
\end{aligned}
$$

where $u, v$, and $w$ are the time-averaged flow velocity components in the $x$-, $y$-, and $z$-direction, respectively; $\varepsilon_{\mathrm{s} x}, \varepsilon_{\mathrm{s} y}$, and $\varepsilon_{\mathrm{s} z}$ are the sediment diffusion coefficients in the $x-, y$-, and $z$-direction, respectively; and $\omega$ is the sediment settling velocity.

In TMR, the crosswise ( $y$-direction) diffusion of sediment from the main flow to the blind channel was completed within a very short distance. For a finite width of blind channel flow, the gravitational action is greatly weakened for sediment transport in the crosswise $(y)$ direction owing to the intense entrainment by larger-scale vortices in TMR. Even if there is some scour-and-fill of sediment, the nonequilibrium of crosswise transport thus caused is also very weak. Therefore, sediment transport in the crosswise direction is approximately in equilibrium in a limited width of TMR. Assuming that the width of the mouth of blind channel is finite, and that the flux of the main flow is much larger than that in the blind channel, the effect of the sediment diffusion from the main flow to the blind channel on the sediment transport in the main flow is weak and negligible, i.e., the sediment entering the blind channel is insignificant compared with the total sediment load carried by the main flow. Consequently, sediment transport in the $x$-direction can be approximately treated as an equilibrium process.

In summary, the sediment transport in TMR can be approximately regarded as an equilibrium process. The action of gravitation in a vertical line is relatively in equilibrium with the turbulent diffusion, so the fifth term at the left side and the third term at the right side of Eq. (30) can be omitted. Ignoring the vertical velocity component, the sediment diffusion equation becomes:

$\frac{\partial S}{\partial t}+u \frac{\partial S}{\partial x}+v \frac{\partial S}{\partial y}=\frac{\partial}{\partial x}\left(\varepsilon_{s x} \frac{\partial S}{\partial x}\right)+\frac{\partial}{\partial y}\left(\varepsilon_{s y} \frac{\partial S}{\partial y}\right)$.

The gradient of velocity $u$ in the $x$-direction is very small, and the sediment turbulent diffusion in the $x$-direction is far less than in the $y$-direction. Considering the steady process of sediment transport, Eq. (31) can be further simplified as

$u \frac{\partial S}{\partial x}+v \frac{\partial S}{\partial y}=\frac{\partial}{\partial y}\left(\varepsilon_{\mathrm{s} y} \frac{\partial S}{\partial y}\right)$.

Now supposing $\varepsilon_{s y}=\beta \varepsilon_{y}$, where $\varepsilon_{y}=l^{2}(\partial u / \partial y)$ is the eddy viscosity for momentum transport, taking $\partial u / \partial y$ as the average gradient along the width of TMR, i.e. $\partial u$ / $\partial y \approx\left(U_{\mathrm{m}}-U_{\mathrm{b}}\right) / b \approx U_{\mathrm{m}} / b,\left(U_{\mathrm{b}} \approx 0\right)$, the average sediment diffusion coefficient in TMR can be expressed as

$\varepsilon_{\mathrm{s} y}=\beta \varepsilon_{y}=\beta c c_{1}^{2} U_{\mathrm{m}} x$.

Substituting Eq. (33) into Eq. (32), the sediment diffusion equation in TMR is rewritten as follows:

$u \frac{\partial S}{\partial x}+v \frac{\partial S}{\partial y}=\beta c c_{1}^{2} U_{\mathrm{m}} x \frac{\partial^{2} S}{\partial y^{2}}$.

The boundary condition of Eq. (34) also can be specified at the outer and the inner boundaries of TMR. In the coordinate system shown in Fig. 3, at the outer boundary $\zeta=\zeta_{1}$ the following relation is obtained:

$S=S_{\mathrm{m}}$,

where $S_{\mathrm{m}}$ is the sediment concentration in the main flow. 
The sediment concentration at the inner boundary can be assumed to be the mean concentration in the circulation flow, $S_{\mathrm{r}}$. Since $S_{\mathrm{r}}$ is unknown in advance, the sediment carrying capacity of the circulation flow can be approximately applied at the inner boundary $\zeta=\zeta_{2}$ :

$S=S_{\mathrm{r}^{*}}$.

where $S_{\mathrm{r}^{*}}$ is the sediment carrying capacity of the circulation flow.

Liu [6] has previously established a formula for estimating the sediment carrying capacity of the circulation flow based on theoretical analysis and experimentation as follows:

$S_{\mathrm{r}^{*}}=K\left(\frac{U_{\mathrm{r} \omega}^{3}}{g B_{0} \omega}\right)^{m}$

where $U_{\mathrm{r} \omega}$ is the intensity of the circulation flow (average velocity on the crosswise axis of circulation flow), $B_{0}$ is the width of blind channel, $\omega$ is the sediment fall velocity, $g$ is the gravity acceleration, $K$ is a coefficient, and $m$ is an exponent.

\subsection{Solution}

Through turbulent mixing, the water flow in TMR transmits not only momentum, but also mass into blind channels, namely, sediment particles carried by water flow. Therefore, the sediment transport also exhibits similarity as does the water flow in TMR. This has been demonstrated by the experiment results as shown in Fig. 6 .

Introducing the similarity function $F(\zeta)$, where $\zeta=\delta y / x$ ( $\delta$ has the same meaning as previously mentioned), the solution to Eq. (34) can be written as

$S(x, y)=S_{\mathrm{m}} F(\zeta)$.

Substituting Eqs. (20), (21) and (38) into Eq. (34) and simplifying it, one obtains

$f(\zeta) F^{\prime}(\zeta)+\beta c c_{1}^{2} \delta^{2} F^{\prime \prime}(\zeta)=0$.

The boundary conditions of Eq. (39) are

$F(\zeta)=1$, at $\zeta=\zeta_{1}$,

$F(\zeta)=S_{\mathrm{r}^{*}} / S_{\mathrm{m}}, \quad$ at $\quad \zeta=\zeta_{2}$.

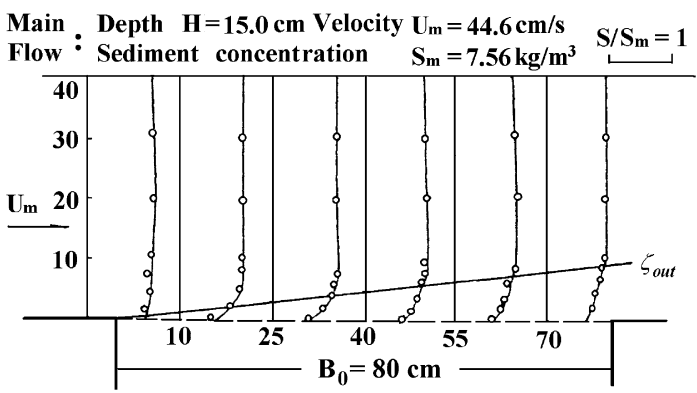

Fig. 6. Distribution across the width of the main flume of sediment concentration near the mouth of the blind channel.
Letting $a=\left(\beta c c_{1}^{2} \delta^{2}\right)^{-1}$, and simplifying Eq. (39) as

$a f(\zeta) F^{\prime}(\zeta)+F^{\prime \prime}(\zeta)=0$.

The general solution of Eq. (42) is

$F(\zeta)=\int_{\zeta_{2}}^{\zeta_{1}} B_{1} \mathrm{e}^{-a \int_{0}^{\varphi} f(\tau) \mathrm{d} \tau} \mathrm{d} \varphi+B_{2}$,

where $B_{1}$ and $B_{2}$ are the integral constants determined by boundary conditions (40) and (41), respectively:

$B_{1}=\frac{\left(S_{\mathrm{m}}-S_{\mathrm{r}^{*}}\right) / S_{\mathrm{m}}}{\int_{\zeta_{2}}^{\zeta_{1}} \mathrm{e}^{-a \int_{0}^{\varphi} f(\tau) \mathrm{d} \tau} \mathrm{d} \varphi}$

$B_{2}=S_{\mathrm{r}^{*}} / S_{\mathrm{m}}$.

Thus, the solution of the sediment diffusion equation in TMR is

$S(x, y)=\frac{S_{\mathrm{m}}-S_{\mathrm{r}^{*}}}{\int_{\zeta_{2}}^{\zeta_{1}} \mathrm{e}^{-a \int_{0}^{\varphi} f(\tau) \mathrm{d} \tau} \mathrm{d} \varphi} \int_{\zeta_{2}}^{\zeta} \mathrm{e}^{-a \int_{0}^{\varphi} f(\tau) \mathrm{d} \tau} \mathrm{d} \varphi+S_{\mathrm{r}^{*}}$.

Defining $g(\varphi)=\int_{0}^{\varphi} f(\tau) \mathrm{d} \tau, g(\varphi)$ can be achieved by integrating Eq. (17):

$$
\begin{aligned}
g(\varphi)= & \int_{0}^{\varphi} f(\tau) \mathrm{d} \tau \\
= & 0.0234 \mathrm{e}^{-A \varphi}-0.8107 \mathrm{e}^{B \varphi} \cos (\sqrt{3} B \varphi) \\
& +0.2378 \mathrm{e}^{B \varphi} \sin (\sqrt{3} B \varphi)+0.7872 .
\end{aligned}
$$

If $a=\left(\beta c c_{1}^{2} \delta^{2}\right)^{-1}$ and $\delta=\left(c c_{1}\right)^{-\frac{2}{3}}$, then $a=\delta c \beta^{-1}$, where $c$ is the coefficient in Eq. (3), namely, $c=\left|\tan \alpha_{1}\right|+\left|\tan \alpha_{2}\right|=$ 0.3179. Consequently,

$a=3.61 \beta^{-1}$,

where $\beta$ is the empirical constant by the Vanoni turbulent exchange hypothesis. Different values of $\beta$ will lead to different values of $a$, and different function $F(\zeta)$. In actual problems, $\beta$ can be determined through comparing computational results and experimental data.

\subsection{Numerical results of sediment diffusion in TRM}

The similarity function $F(\zeta)$ includes two transcendental integral functions, it can be calculated by numerical integration instead of an analytical approach. Based on the sediment diffusion characteristics and laboratory data, $\beta=1.4$ and $a=2.578$, thus, one has

$$
\begin{aligned}
& \int_{\varsigma_{2}}^{\varsigma_{1}} B_{1} \mathrm{e}^{-a g(\varphi)} \mathrm{d} \varphi=2.204, \\
& S(x, y)=0.454\left(S_{\mathrm{m}}-S_{\mathrm{r}^{*}}\right) \int_{-2.403}^{\zeta} \mathrm{e}^{-2.578 g(\varphi)} \mathrm{d} \varphi+S_{\mathrm{r}^{*}} .
\end{aligned}
$$

Then, the concentration distribution in TMR was numerically calculated with a space step $\Delta \zeta=0.2$. Fig. 7 illustrates the comparison of the numerical results (solid line) and the experimental data (dots), which shows a good agreement. The deviation between measured and computed results becomes a little larger near the downstream wall of the blind 


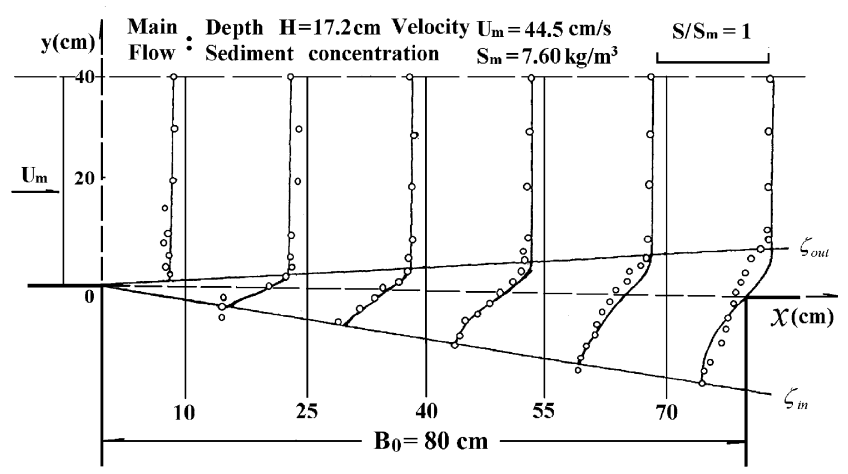

Fig. 7. Distribution of sediment concentration in TRM where the solid lines are numerical results and circles are experimental measurements.

channel because of the wall effects. The deviation also is larger near upstream of the mouth due to the initial development of turbulence. As a whole, simulation results in most regions satisfactorily agree with the real situation, except in the neighborhood regions of the upstream and downstream walls.

\subsection{Gradient of sediment concentration in TMR}

The sediment concentration in TMR exhibits a large gradient along the crosswise axis ( $y$-direction), which causes constant diffusion of sediment from the main flow into the blind channel. The crosswise gradient of the sediment concentration in TMR can be obtained:

$\frac{\partial S}{\partial y}=\delta x^{-1} S_{\mathrm{m}} F^{\prime}(\zeta)$

where

$F^{\prime}(\zeta)=B_{1} \mathrm{e}^{-a g(\zeta)}$,

$B_{1}=0.458\left(1-S_{\mathrm{r}^{*}} / S_{\mathrm{m}}\right)$.

At the mouth of the blind channel $(\zeta=0), F^{\prime}(\zeta)=B_{1}$. Substituting Eqs. (52) and (53), and $\delta=11.29$ into Eq. (51), the concentration gradient at the geometrical interface of blind channels can be obtained as

$$
\left(\frac{\partial S}{\partial y}\right)_{0}=5.17 x^{-1}\left(S_{\mathrm{m}}-S_{\mathrm{r}^{*}}\right)
$$

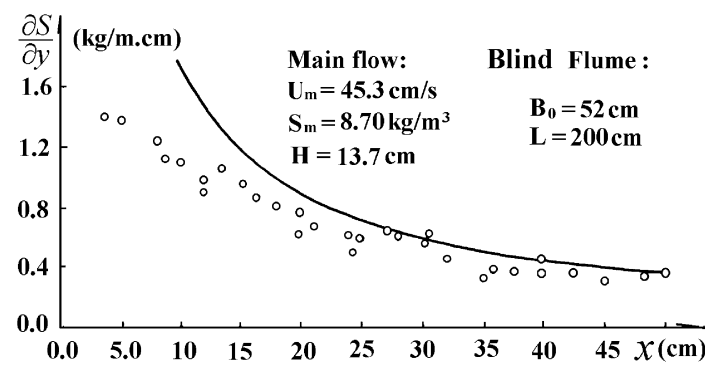

Fig. 8. The gradient of sediment concentration on the geometrical interface $(\zeta=0)$ computed from Eq. (54) (solid line) and experimentally measured.
Comparison of the numerical results of Eq. (54) and the experimental data is shown in Fig. 8. The experimental values of sediment concentration gradient were obtained by computing the average variation of sediment concentration from a point $1 \mathrm{~cm}$ on the main-flow side to another point $1 \mathrm{~cm}$ on the circulation flow side. Therefore, the experimental results are systematically smaller than the computational values. However, this comparison still shows that Eq. (54) may describe well the variation of the sediment concentration gradient on the interface.

\section{Estimation of the sediment deposition rate in blind channels}

There exists a circulation flow with a vertical axis near the mouth of blind channels. Because of low sediment-carrying capacity for circulation flow, a large amount of sediment deposition occurs in this region. In general, there exits turbulent diffusion and convection between the main flow and the blind channel flow according to Eqs. (31) and (32). All sediment in blind channel comes from the main flow through turbulent diffusion and convection in TMR. For general engineering projects, the circulation flow in blind channels is very weak, i.e., the velocities in the circulation flow are very low. The circulation flow in the blind channel could be approximately considered a closed flow induced by the main flow [11]. For this situation, the convection between the main flow and the blind channel flow is very weak and negligible. Ignoring the complex processes of sediment transport and deposition in the circulation flow region, it is assumed that all of the sediment particles entering the blind channel will ultimately deposit there. Consequently, the sediment deposition rate in the blind channel can be obtained by integrating the sediment diffusion rate crossing the interface between the main flow and the blind channel flow.

The sediment diffusion rate crossing a unit area of the interface can be expressed by Eq. (1), i.e. $g_{\mathrm{s}}=\varepsilon_{\mathrm{s} y}(\partial S / \partial y)$ at the interface. For a finite width of blind channel, the actual interface between the main flow and the circulation flow is approximately coincidental with the geometrical interface. Therefore, integrating along the geometrical interface $(y=0)$ instead of the actual interface, the sediment amounts entering into the blind channel per unit time, $G_{\mathrm{s}}$, can be derived as follows:

$G_{\mathrm{s}}=H \int_{0}^{B_{0}} \varepsilon_{\mathrm{s} y} \frac{\partial S}{\partial y} \mathrm{~d} x$

where $H$ is the water depth, $B_{0}$ is the width of the blind channel.

In general, the water depth in the blind channel should decrease with the time because of sediment deposition. However, the sediment deposition mainly take places in the circulation region, the water depth at the mouth of blind channels commonly varies slowly in most practical engineering projects. Consequently, the water depth, $H$, 


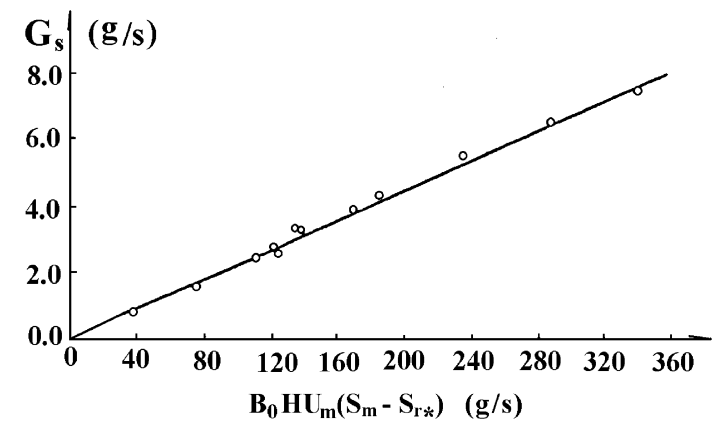

Fig. 9. Comparison of sediment deposition rate in blind channels computed by Eq. (56) (solid line) and experimental data (circles).

can be assumed as a constant in a finite period. Substituting Eqs. (33) and (54) into Eq. (55), finally, the following can be obtained:

$G_{\mathrm{s}}=0.0235 U_{\mathrm{m}} B_{0} H\left(S_{\mathrm{m}}-S_{\mathrm{r}^{*}}\right)$.

Eq. (56) describes the total amount of sediment entering the blind channel per unit time, i.e., the total amount of sediment deposition in the blind channel per unit time, which is the so-called the sediment deposition rate in the blind channel. Eq. (56) was well validated by experimental data as shown in Fig. 9. The results indicate that Eq. (56) established in this paper can correctly estimate the sediment deposition rate in blind channels.

Eq. (56) reveals, for a low velocity flow system, that the sediment deposition rate in blind channels is generally proportional to the velocity of the main flow, water depth, the width of the blind channel, and the difference in sediment concentration between the main flow and the circulation flow in the blind channel. However, when the velocity of the main flow increases to certain great value, the sediment deposition rate may decrease with the increase of the velocity of the main flow. The enough main flow velocity can cause the obvious increase of circulation intensity and sediment concentration in blind channels, which should lead to the obvious decrease of the difference in sediment concentration between the main flow and the blind channel flow. This law was also supported by the experimental observations of $\mathrm{Xu}$ [12].

In practical application, as the generally very small flow velocity and sediment concentration of flow in blind channels, the sediment concentration in blind channels can be approximated as zero. Consequently, Eq. (56) further simplifies to the following form:

$G_{\mathrm{s}}=0.0235 U_{\mathrm{m}} B_{0} H S_{\mathrm{m}}$.

\section{Conclusions}

The properties of the water flow and sediment diffusion at the mouth of finite width of blind channels were experimentally and theoretically investigated, and the following conclusions are drawn from this study:
(1) The turbulent mixing region (TMR) between the main flow and the circulation flow in a blind channel is an important area where the main flow continually transfers momentum and sediment into the circulation flow. The accumulative deposition of suspended sediment in the blind channel is governed by turbulent diffusion in TMR.

(2) Experimental data show that the water flow and sediment motion approximately coincide with the similarity structure in TMR. The similarity solutions of flow motion and sediment diffusion were finally obtained by theoretical analysis.

(3) The distributions of longitudinal velocity and sediment concentration in TMR were numerically calculated. Comparisons of numerical results and experimental data show rather good agreement.

(4) A formula (Eq. (56)) to estimate the sediment deposition rate in blind channels with the finite width was theoretically established based on the sediment diffusion in TMR. The fact that the sediment carrying capacity in the circulation flow has a significant influence on the sediment diffusion between the main flow and the circulation flow is effectively considered.

\section{Acknowledgements}

This research is financially supported by the Key Projects of the Chinese Natural Science Foundation (No. 10332050) and the Open Research Fund Program of the State Key Laboratory of Water Resources and Hydropower Engineering Science, Wuhan University, China.

\section{References}

[1] Altai W, Shafie M, Chu VH. Recirculating flow in a shallow basin. Proc. 29th IAHR congress. Theme D, vol. 1. Beijing: Tsinghua University Press; 2001. p. 52-7.

[2] Dong YH. Calculation and analysis on flow and sediment transport in cavity circulation. J Sediment Res 1999;2:34-9 (in Chinese).

[3] Hangen RL, Dhanak AM. Momentum transfer in turbulent separated flow past a rectangular cavity. J Appl Mech ASME 1966;33(3):641-6.

[4] Hofland B, Christiansen H, Crowder RA, Kirby R, van Leeuwen $\mathrm{CW}$, Winterwerp JC. The current deflecting wall in an estuarine harbour. Proc. 29th IAHR congress. Theme D, vol. 1. Beijing: Tsinghua University Press; 2001. p. 613-21.

[5] Mizumura K, Yamamoto T, Fujikawa T. Prediction of sand movement bear port of Kanazawa. J Waterway, Port, Coastal, Ocean Eng, ASCE 1997;123(5):215-22.

[6] Landau LD, Lifshitz EM. Fluid Mechanics. 2nd ed. Course of theoretical physics. vol. 6, 157-189. Reprinted by Beijing World Publishing Corporation by arrangement with Butterworth-Heinemann (A Division of Reed Educational and Professional Publishing Ltd.), 1999.

[7] Liu QQ. Saturated sediment carrying capacity of circulating flow. Int J Sediment Res 1999;14(3):45-51.

[8] Liu QQ. Experimental study on flow and sediment movement in cavity circulations. Int J Sediment Res 2002;17(2):147-56.

[9] van Schijndel SAH. Reducing the siltation of a river harbour. J Hydraul Res 1998;36(5):803-14.

[10] Schmidt JC. Recirculating flow and sedimentation in the Colorado River in Grand Canyon, Arizona. J Geol, AGU 1990;98:709-24. 
[11] Xie JH, Yin RL. Sedimentation problems of waterway leading to navigation in low water level hinge engineering. Proc of the second international symposium on river sediment. Beijing: Chinese Hydraulic and Electric Press; 1983.

[12] Xu JY. Experimental studies on the mechanism of water and sediment movement of circulation flow at the mouth of a blind channel. Master's Thesis, Wuhan University of Hydraulic and Electric Engineering. China. 1982 (in Chinese).

[13] Yue JP. Experimental study on the sediment deposition caused by rotating flow in the closed channel. J Sediment Res 1986;2:41-50 (in Chinese). 